\title{
Efficacy and Safety for the Combination of
}

\section{Levosalbutamol, Ambroxol and Guaifenesin for the Symptomatic Treatment of Productive Cough: Phase IV Clinical Trial}

\author{
Dr. Mayuresh Dilip Kiran ${ }^{* 1}$, Lalit Jeevan Pawaskar², Shaheen Naseem Sheikh ${ }^{3}$ \\ ${ }^{1}$ Vice President, Medical Services, ${ }^{2}$ Executive, Pharmacovigilance, ${ }^{3}$ Research Associate, Pharmacovigilance, \\ Centaur Pharmaceuticals Pvt. Ltd
}

\section{Corresponding Author -}

\section{Dr. Mayuresh Kiran}

Centaur Pharmaceuticals Pvt. Ltd.,

Centaur House, Near Grand Hyatt, Vakola, Santacruz (East), Mumbai - 400055

Email: mayuresh_kiran@rediffmail.com

\begin{abstract}
Background: Cough is one of the most common symptom that most of the Doctors faces while practising in paediatric population. Cough can be productive or unproductive. Productive cough can be treated with the combination of bronchodilator, expectorant and mucolytics. The main objective of the Phase IV clinical trial conducted was to test the efficacy and safety for the combination of Levosalbutamol (bronchodilator), Ambroxol Hydrochloride (mucolytic agent) and Guaifenesin (expectorant) for the treatment of productive cough associated with bronchospasm in conditions such as bronchitis and bronchial asthma as well as all conditions associated with tenacious mucus, wheezing and chest congestion. Methodology: The trial was conducted with 23 paediatric speciality Doctors on 325 patients out of which 149 patients were of age group 2 to 12 years and 176 were of age below 1 year. Efficacy assessment was done by analysing the cough severity score at all the visits. Safety assessment was done by analysing the adverse events experienced by the patients or observed by the investigators at all the visits. $\underline{\boldsymbol{R} e s u l t s:}$ At baseline, CSS was 6.624 reduced to 3.744 at day 3 and 1.049 at day 5. One point reduction in Likert type symptom scale from moderate to mild was in just 5 days. Only $12.923 \%$ of patients experienced non-serious adverse events. Conclusion: Combination of Levosalbutamol, Ambroxol Hydrochloride and Guaifenesin was safe as well as efficacious for the treatment of productive cough.
\end{abstract}

Keywords: Levosalbutamol, Ambroxol, Guaifenesin, Cough

\section{Introduction}

Cough is one of the most common symptom that paediatricians faces while practising. According to duration of the cough it can be acute or chronic, recidivist, recurrent or persistent. Cough can be productive or unproductive. Productive cough is the cough with respiratory tract secretions and unproductive cough is the cough with no respiratory tract secretions. A productive cough produces phlegm or mucus or sputum. ${ }^{[1.2]}$ Productive cough leads to expectoration of sputum for the duration of more than 8 weeks. ${ }^{[3]}$ Productive cough is a symptom which is mostly observed in developing countries. In developing countries incidence of productive cough is about 20 to $30 \%$ and in developed countries it is 3 to $4 \%$. $^{[4]}$

According to A Vora et al productive cough can be treated with the combination of bronchodilators, expectorants and mucolytic agents. ${ }^{[5]}$ This PMS study was conducted to test the safety and efficacy for the combination of bronchodilators (Levosalbutamol), mucolytic agents (Ambroxol Hydrochloride) and expectorant (Guaifenesin) for the treatment of productive cough in the children patients of different age group, including age up to 1 year and 2 to 12 years for the treatment of productive cough associated with bronchospasm in conditions such as bronchitis and bronchial asthma as well as all conditions associated with tenacious mucus, wheezing and chest congestion.

Levosalbutamol activates the adenyl cyclase and lowers the intracellular concentration of 3'5'-cyclic AMP (cAMP). Bronchial smooth muscles in the airways gets relaxed because of increased concentration of cAMP by reducing intracellular calcium level and prevents concentration of hyper-responsive airways. Increased concentration of cAMP also inhibits the release of eosinophils and inflammatory mediators from mast cells. Levosalbutamol interacts with $\beta 2$ adrenoreceptors and has anti-edematous, bronchodilator and bronchoprotective properties and inhibits activation of 


\section{International Journal of Innovative Research in Medical Science (IJIRMS) Volume 04 Issue 01 Jan 2019, ISSN: 2455-8737, Imp. Factor - 4.102 \\ Available online at $-\underline{w w w . i j i r m s . i n}$}

eosinophils and mast cells. Levosalbutamol has approximately 100 and 2 times greater binding affinity for $\beta 2$ adrenergic receptor than S Salbutamol and racemic salbutamol respectively. ${ }^{[6]}$

Ambroxol hydrochloride is a mucolytic agent, it also increases bronchial secretion and reduces the viscosity of tenacious sputum and it expedites the removal of it by coughing. Ambroxol hydrochloride also upturns the clearance of mucociliary secretions. ${ }^{[7,8]}$

Guaifenesin is an expectorant which increases the amount of output of bronchial secretions and sputum by reducing the viscosity of the secretions. It reduces the surface tension and adhesiveness of sputum. The improved flow of bronchial secretions endorses ciliary action which changes an unproductive, dry cough to less frequent and more productive. By reducing the adhesiveness and viscosity of sputum, It increases the efficacy of cough reflex, ciliary actions and mucociliary removal for accumulated bronchial secretions from the lower and upper airway. ${ }^{[9]}$

\section{Methodology}

This was a Phase IV Clinical trial conducted with 23 Paediatric speciality investigators from different cities all across the India. The clinical trial was started in May 2017 and completed in October 2017. Total 380 patients were recruited for the study out of which 325 patients completed and 54 patients were lost to follow-up. Out of 325 patients 149 patients were of age group 2 to 12 years and 176 patients were of age group up to 1 year.

\section{Inclusion and Exclusion criteria}

Patients with confirmed diagnosis of productive cough associated with bronchospasm in conditions such as bronchial asthma and bronchitis as well as all conditions associated with tenacious mucus, chest and wheezing congestion were enrolled in the study. Patients of both the genders (male as well as female) having age up to 12 years and weight up to $48 \mathrm{Kg}$ were recruited for the study. Finally the patients who were ready to strictly follow and adhere to the protocol and sign informed consent form (ICF) were recruited for the study.

Patients having hypersensitivity to any individual study drug of the study drug combination or to any of the excipient present in the dosage form and patients suffering from hepatic or renal dysfunction and patients who cannot adhere to the protocol i.e. mentally ill and patients with psychological problem were excluded.

\section{Sample Size}

The minimum sample size was decided to be kept 300 patients and by considering the loss of approximately 80 patients total 380 patients were recruited for the study out of which 54 patients were lost to follow-up and the total study was completed with 325 patients.

\section{Study Intervention}

A study drug combination of Levosalbutamol, Ambroxol Hydrochloride and Guaifenesin was used for the phase IV clinical trial. Different dose combination in different dosage forms were used for the infants and children. For infants of age up to 1 year, the combination of Levosalbutamol $0.25 \mathrm{mg}$, Ambroxol $7.5 \mathrm{mg}$,
Guaifenesin $12.5 \mathrm{mg}$ and mentholated flavoured syrupy base quantity sufficient per $\mathrm{ml}$ was given in the form of oral drops dosage form and for children of age more than 2 years and up to 12 years the combination of Levosalbutamol sulphate equivalent to Levosalbutamol $1 \mathrm{mg}$, Ambroxol hydrochloride $30 \mathrm{mg}$, Guaifenesin $50 \mathrm{mg}$ and Mentholated flavoured syrupy base quantity sufficient per $5 \mathrm{ml}$ was given in the syrup dosage form.

Guardians of the patients recruited of age up to 1 year in the study were given $10 \mathrm{ml}$ free physician sample of study drug combination drops. Guardians of children patients of age 2 to 12 years recruited in the study were given 2 bottles of $50 \mathrm{ml}$ free physician sample of study drug combination syrup. Guardians will be advised to give it to the patients in the dose as per the following table for the clinical trial duration of 5 days.

Table 1: Dose of study drug combination syrup and oral drops to be given to the patients of different age group

\begin{tabular}{|c|c|c|c|}
\hline Age & Body weight & dose & $\begin{array}{c}\text { Dosage form of } \\
\text { study drug } \\
\text { combination }\end{array}$ \\
\hline Up to 1 years & Up to $11.8 \mathrm{~kg}$ & $2.5 \mathrm{ml}$ tid & Oral Drops \\
\hline $2-5$ years & $2-21 \mathrm{~kg}$ & $5 \mathrm{ml}$ tid & Syrup \\
\hline $6-12$ years & $18-48 \mathrm{~kg}$ & $10 \mathrm{ml} \mathrm{tid}$ & Syrup \\
\hline
\end{tabular}

\section{Study procedure}

The trial was conducted for the duration of 5 days. Patients who were suffering from productive cough associated with bronchospasm in conditions such as bronchitis and bronchial asthma as well as all the conditions associated with tenacious mucus, wheezing and chest congestion who met with the inclusion and exclusion criteria were recruited for the phase IV clinical trial. A detailed medical history was obtained and also physical examination was conducted for each patient recruited for the study by the investigators.

The study was conducted in 2 groups of patients, in $1^{\text {st }}$ group, patients of age up to 1 year and in $2^{\text {nd }}$ group the patients recruited were of age 2 to 12 years. For both groups of patients, the investigators selected were of paediatric speciality from all over the India. Patients of group 1 and group 2 were treated with the study drug combination, oral drops and syrup as per the table 1 for the clinical trial duration of 5 days.

All the guardians of the patients recruited for the study were instructed to maintain a diary of all the adverse events occurring during the study period. Three visits were planned for all the patients, the first visit was baseline visit (V1) on day 1 in which physical examination was done before treating the patient with the study drug combination. The second visit was revaluation visit (V2) on day 3 and third visit (V3) was on day 5. Efficacy assessment was done with the help of cough severity scale and cough severity score (CSS) was recorded at all the visits. And for the assessment of safety the adverse events experienced by the patient or observed by the clinical trial investigator i.e. Dr was recorded. Investigators were asked to discontinue the study drug in case of severe adverse event and with discretion, clinical experience in case of mild or moderate adverse events. All the efficacy parameters and adverse events experienced by the patient or observed by the investigator were recorded in the case record form by the investigator, collected at the end of the trial and analysed. 


\section{Concomitant therapy}

No Pharmacological intervention including any drug or drug combination other than study drug combination was allowed to take during the clinical trial duration of 5 days. NonPharmacological interventions like drinking of hot water at regular intervals or steam inhalation were allowed and encouraged during the study period.

\section{Efficacy Assessment}

The primary assessment was done by evaluating the reduction in CSS of all the symptoms related to cough on an eleven point scale. CSS is recorded with the help of cough severity scale which is an 11 point scale in which 0 is no symptoms and 10 means maximum symptoms. CSS scale was further extrapolated to Likert-type symptom severity scale where 0 on CSS scale means no symptoms, 1 to 3 means mild symptoms, 4-6 means moderate symptoms and 7-10 means severe symptoms.

In primary efficacy assessment decrease in mean CSS of all the patients at each visit, percentage decrease in CSS at visit 2 and 3 as compared to baseline was calculated. In secondary efficacy assessment percentage of patients having CSS of mild, moderate and severe intensity was calculated and presented graphically.

\section{Safety assessment}

Throughout the clinical study, investigators asked patients for any adverse events and if found then it was noted in the case report form (CRF) during each post-dose visit. Noted adverse events were classified into 2 categories as serious or non-serious adverse events. Adverse events were classified as drug related or nondrug related adverse events by using Naranjo's scale of probability. Adverse events observed were followed up and treated if necessary by the investigators till their resolution.

\section{Regulatory and Ethical matters}

The study drug combination is currently available in India and classified under schedule $\mathrm{H}$ drugs i.e. it can be sold only in the presence of a prescription of a registered medical practitioner. All the patients recruited in the study have read informed consent form and signed the same. Clinical trial protocol, ICF, CRF, undertaking by the investigators form, ethical committee certificates were collected before initiating the clinical study.

\section{Results}

Total 380 patients were recruited at 23 centers across India, 325 patients completed the study. Demographic characteristics are mentioned in the table 2 .

Table 2: Demographic characteristics of the patients recruited in the clinical trial

\begin{tabular}{|l|c|}
\hline Mean age of patients (years) & 2.89 years \\
\hline No. of males patients & 189 \\
\hline No. of females patients & 136 \\
\hline
\end{tabular}

\section{Efficacy analysis}

At baseline i.e. visit 1 the mean CSS score was 6.624 which was reduced to 3.744 after initiating the treatment with the study drug combination at visit 2 i.e. at day 3 and it was further reduced to 1.049 at visit 3 i.e. at day 5. Mean CSS at all the visits is presented graphically at figure 1 .

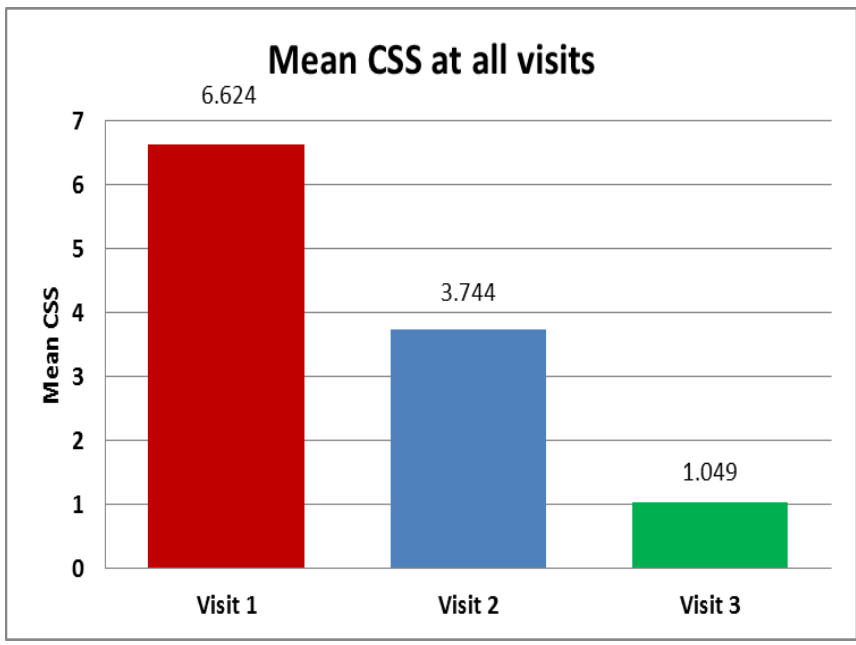

Figure 1: Mean CSS score at all the visits

As compared to baseline at visit 2 and visit 3 there was reduction of $43.478 \%$ and $84.183 \%$ at visit 2 and visit 3 respectively. Percentage decrease in mean CSS at visit 2 and 3 is presented graphically in figure 2.

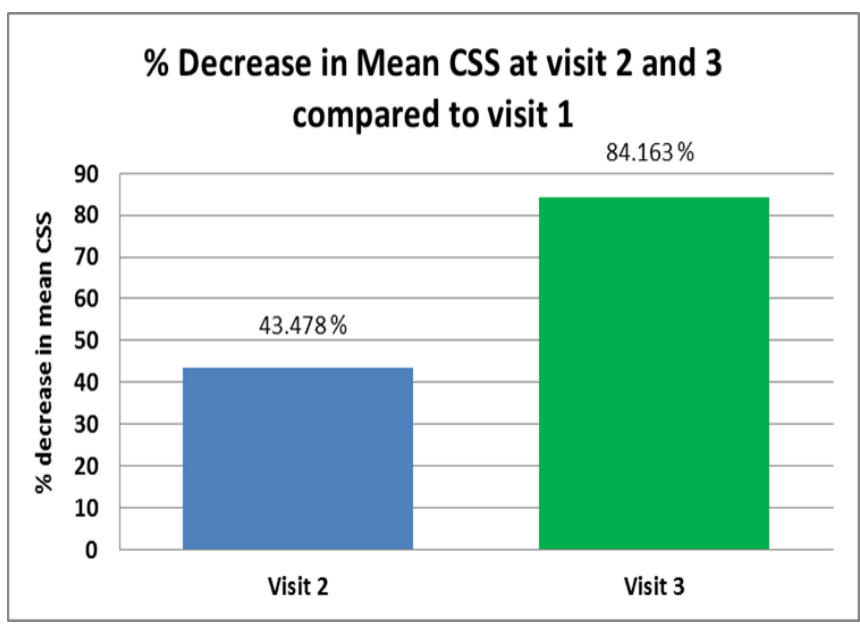

Figure 2: Percentage decrease in mean CSS at visit 2 and visit 3 as compared to baseline

CSS scale was further extrapolated to Likert-type symptom severity scale. At visit 1;170, 134 and 21 patients were of severe, moderate and mild intensity of CSS respectively. At visit 2; 3, 192 and 126 patients were of severe, moderate and mild intensity of CSS and 4 patients were having no symptoms. At visit 3; 21 and 176 patients were of moderate and mild intensity of CSS. And at visit 2 and visit 3; 4 and 128 patients respectively had 0 CSS. All the patients having different CSS at visit 1,2 and 3 is presented graphically in figure 3 . 


\section{International Journal of Innovative Research in Medical Science (IJIRMS) Volume 04 Issue 01 Jan 2019, ISSN: 2455-8737, Imp. Factor - 4.102
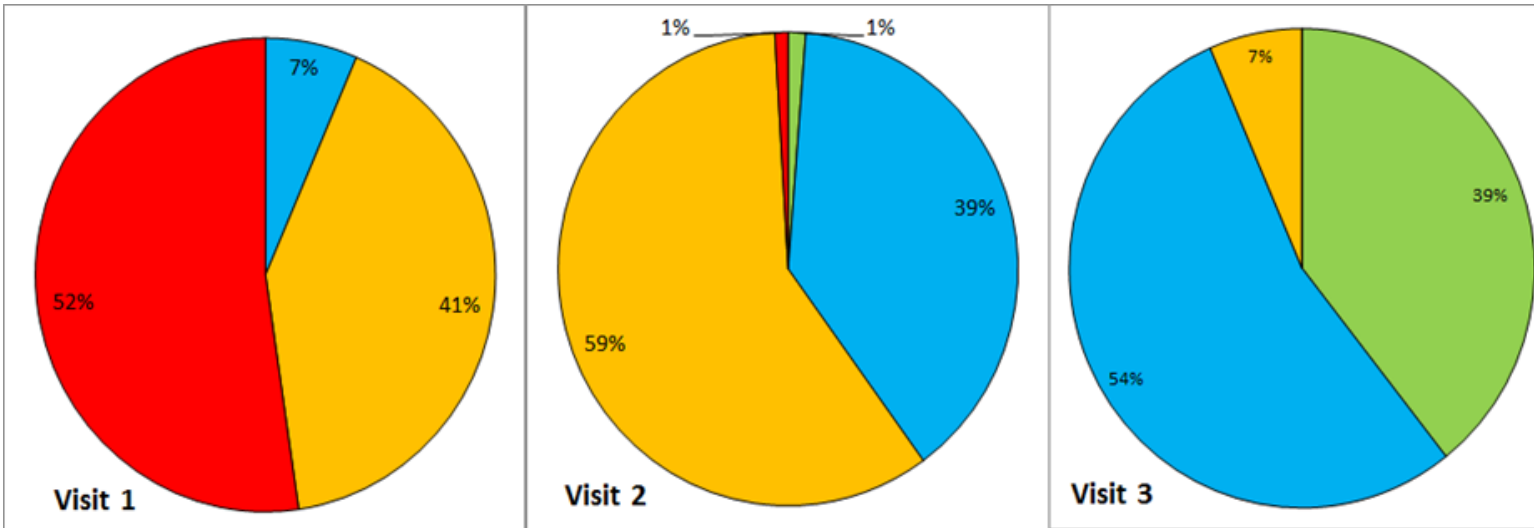

$\square$ No symptoms

$\square$ Mild symptoms

Visit 2

Visit 3

口Moderate symptoms

Figure 3: Patients having none, mild, moderate and severe CSS at visit 1, 2 and 3

\section{Safety analysis}

The overall incidences of reported study drug combination related adverse events were seen in 61 patients. The list of adverse events with the number of episodes is mentioned in Table 3.

Table 3: Adverse events, no. of episodes, no. of patients and percentage of patients experienced from total population

\begin{tabular}{|l|c|c|c|}
\hline $\begin{array}{l}\text { Adverse } \\
\text { Events }\end{array}$ & $\begin{array}{c}\text { No. of } \\
\text { episodes }\end{array}$ & $\begin{array}{c}\text { No. of } \\
\text { patients }\end{array}$ & \% of patients \\
\hline Dizziness & 54 & 31 & $9.538 \%$ \\
\hline Nausea & 21 & 10 & $3.076 \%$ \\
\hline Heartburn & 38 & 23 & $7.076 \%$ \\
\hline Diarrhea & 32 & 15 & $4.615 \%$ \\
\hline Total & 145 & 61 & $12.923 \%$ \\
\hline
\end{tabular}

\section{Discussion}

According to A Vora et al productive cough can be treated by the combination of bronchodilators, expectorants and mucolytics. ${ }^{[5]}$ This PMS study was conducted to test the efficacy as well as safety for the combination of Levosalbutamol, Ambroxol and Ambroxol Hydrochloride for the treatment of productive cough associated with bronchospasm in conditions such as bronchitis and bronchial asthma as well as all conditions associated with tenacious mucus, wheezing and chest congestion in patients of age up to 12 years.

At baseline mean CSS score was 6.624 and after initiating the treatment the mean CSS was reduced to 3.744 at visit 2 which was further reduced to 1.049 at visit 3 . At visit 2 the mean CSS score was reduced by $43.478 \%$ which was further reduced to $84.163 \%$ at visit 3 as compared to baseline. So the study drug combination was efficacious in reducing the CSS within 5 days. When the CSS score was extrapolated to likert scale then it was found that at visit 1, 170 patients were having severe symptoms of productive cough, 134 patients were having moderate symptoms and 21 were having mild symptoms. At visit 2 the number of patients having severe symptoms of productive cough were reduced to 3 which was 170 in number at baseline and the number of patients having moderate and mild symptoms were 192 and 126 respectively. At visit 2, 4 patients were completely cured from the productive cough symptom. At visit 3, no patient was having severe symptoms of productive cough, only 21 patients of moderate symptoms, 176 patients of mild symptoms and 128 patients were completely got symptomatic relief from productive cough. So at visit 3 total 39.38 $\%$ of patients got total symptomatic relief from the productive cough and $54.15 \%$ of patients were having mild symptoms.
Mostafa Yakoot, Amel Salem and Abdel Mohsen Omar conducted the pragmatic, randomized, single blinded, comparative study to determine the efficacy in reducing symptom of cough, sputum and breathlessness. For study both male and female patients were shortlisted of age less than 45 years. Patients having history of smoking, patients diagnosed with acute cerebrations and chronic bronchitis were recruited for the study. Total 100 patients were randomized to receive either the combination of Ambroxol (15 $\mathrm{mg}$ ); Theophylline (50 mg); Guaifenesin (30 mg), per $5 \mathrm{ml}$ syrup or Guaifenesine $(100 \mathrm{mg})$ per $5 \mathrm{ml}$. In addition patients were received ciprofloxacin $500 \mathrm{mg}$, twice daily, for 7 days); inhaled or nebulized bronchodilator, salbutamol (two inhaler puffs or $5 \mathrm{mg}$ in $2 \mathrm{~mL}$ saline nebulized); and/ or ipratropium $0.5 \mathrm{mg}$ as needed, or up to four times daily or nebulized salbutamol. Breathlessness was measured on a 7-point Likert scale. Severity of cough and sputum clearance was measured using the Modified Questionnaire for Ease of Cough and Sputum Clearance. At the end of one week treatment, all patients were asked to grade their impression of improvement or change in their condition using the Clinical Global Impression of Improvement or Change Scale (CGIC), 11 which is a 7-point rated scale where $0=$ not assessed; $1=$ very much improved; 2 = much improved; 3 = minimally improved; $4=$ no change; $5=$ minimally worsened; $6=$ much worsened; and $7=$ very much worsened. Baseline score for breathlessness for both study combination and Guaifenesin syrup was 4 which was reduced to 2 and 3 at day 3 for combination and single Guaifenesin syrup respectively. At day 7, score for breathlessness was further reduced to 1 and 2 for combination and single Guaifenesin syrup respectively. At baseline the cough score for both the groups was 16 which was reduced to 10 and 13 at day 3 for study combination and Guaifenesin respectively. At day 7 the cough score was further reduced to 8 and 12 for study combination and Guaifenesin respectively. So in the study duration it was found that study drug combination of Ambroxol (15 mg); Theophylline (50 mg); Guaifenesin $(30 \mathrm{mg})$, per $5 \mathrm{~mL}$ syrup was more efficacious than Guaifenesin (100 mg) per $5 \mathrm{ml}$ for reducing the breathlessness and cough score. ${ }^{[8]}$

Kurli. Sankar et al conducted a parallel, comparative and prospective clinical trial to evaluate and compare the efficacy and adverse events causing by Salbutamol and Levosalbutamol. The study was conducted on total 210 patients and 105 patients in each group. One group was treated with $2 \mathrm{mg}$ racemic Salbutamol 3 times a day and second group was treated with $1 \mathrm{mg}$ Levosalbutamol given orally thrice a day. Both the groups were treated for the study duration of 21 days. FEV1 was measured at baseline and after every 7 days as an efficacy evaluation parameter 
and results were analysed statistically. At baseline FEV1 and \% FEV1 for Salbutamol group was 1.54 and $76.51 \%$ which was increased to 1.58 and $78.4 \%$ at the end of the study and at baseline FEV1 and \% FEV1 for Salbutamol group was 1.66 and $75.29 \%$ which was increased to 1.72 and $78.43 \%$ at the end of the study. The adverse events experienced by the patients because of Levosalbutamol were significantly low as compared to Salbutamol. Paired t test was used to compare the FEV1 and \% FEV1 value before and after the study period in each group separately. In which $\mathrm{T}$ value of FEV1 and \%FEV1 was 23.6956 and 23.6332 and $\mathrm{P}$ value of FEV1 and \%FEV1 was $<0.0001$ and $<0.0001$. Improvements in FEV1 and \%FEV1 values were statistically significant in Salbutamol and Levosalbutamol group. Finally it was concluded that both the drugs are efficacious but Levosalbutamol was more superior to salbutamol in terms of safety and tolerability. ${ }^{[10]}$

\section{Conclusion}

Combination of Levosalbutamol $0.25 \mathrm{mg}$, Ambroxol Hydrochloride $7.5 \mathrm{mg}$ and Guaifenesin $12.5 \mathrm{mg}$ per $\mathrm{ml}$ and combination of Levosalbutamol $1 \mathrm{mg}$, Ambroxol hydrochloride 30 $\mathrm{mg}$ and Guaifenesin $50 \mathrm{mg}$ per $5 \mathrm{ml}$ is safe as well as efficacious for the treatment of productive cough associated with bronchospasm in conditions such as bronchitis and bronchial asthma as well as all conditions associated with tenacious mucus, wheezing and chest congestion in the paediatric population of age up to 1 year and of age 2 to 12 years respectively.

\section{Acknowledgement}

We would like to acknowledge Dr. Makarand Shinde (Borivali), Dr. Mamota Sampathy (Cuttack), Dr. Nilakantha Sahu (Angul), Dr. Chetna Parekh (Kandivali), Dr. Mahesh Holeyannavar (Hobli), Dr. Palakesh Mundur (Balgaum), Dr. Sachin Kinnarkar (Hubli), Dr. Sachin Sagavkar (Ichalkaranji), Dr. Prashant Kumar Das (Mayurbhanj), Dr. Shyam Sagar (Gadhirganj), Dr. Vijay shinde (Karjat), Dr. Swati Shrotri (Satara), Dr. Binaya Kumar Nath (Angul), Dr. Mahesh Kumar Mohanti (Keonjhar), Dr. Nitin Kokare (Kalyan), Dr. Rabindra Kumar Nanda (Jajpur Town), Dr. Vinayak Mahanavar (Sangli), Dr. Vijay Tejam (Kolhapur), Dr. Jyoti Mhaske (Solapur), Dr. Digvijay Maralkar (Kolhapur), Dr. Chandramohan Hajari (Jogeshwari), Dr. Gopal Chandra Deka (Jorhat), Dr. Santosh Vajrashetty (Kanjurmarg) and Dr. Palash Hazrika (Naigaon) who were the co-investigators in this study.

\section{Disclosure}

This study was conducted as a part of Pharmacovigilance activity for Kofarest PD Drops and Kofarest LS Syrup of Centaur Pharmaceuticals Pvt. Ltd.

\section{Conflict of Interest - None}

\section{Reference}

[1] Begic E, Begic Z, Dobraca A, Hasanbegovic E. Productive Cough in Children and Adolescents-View from Primary Health Care System. Medical Archives. 2017 Feb;71(1):66.

[2] Chung KF, Widdicombe JG, Boushey HA, editors. Cough: causes, mechanisms and therapy. John Wiley \& Sons; 2008 Apr 15.

[3] Martin, Matthew J., and Tim W. Harrison. "Causes of chronic productive cough: an approach to management." Respiratory medicine 109.9 (2015): 1105-1113.

[4] Vijay S, Dalela G. Prevalence of LRTI in patients presenting with productive cough and their antibiotic resistance pattern. Journal of clinical and diagnostic research: JCDR. 2016 Jan;10(1):DC09.

[5] Vora A, Bhargava A. A Cross-sectional Cohort Analyses Assessing Response to Levosalbutamol Bronchodilatory Cough Formulations in Outpatient Community Settings of India:'BUS'analyses. The Journal of the Association of Physicians of India. 2016 Sep;64(9):45-8.

[6] Mukesh Kumar Gupta, Meenu singh. Evidence based on review on Levosalbutamol. Indian Journal of Pediatrics. 2007;74:161-167.

[7] Tripathi KD. Essentials of medical pharmacology. JP Medical Ltd; 2013 Sep 30.

[8] Yakoot M, Salem A, Omar AM. Clinical efficacy of farcosolvin syrup (ambroxol-theophylline-Guaifenesin mixture) in the treatment of acute exacerbation of chronic bronchitis. International journal of chronic obstructive pulmonary disease. 2010;5:251.

[9] https://www.accessdata.fda.gov/drugsatfda_docs/nda/200 2/21-282_Mucinex_biopharmr.pdf last viewed on 15 June 218.

[10] Ch J, Kambar C, Kurli S. Comparative study of efficacy and adverse effects profile of telmisartan vs enalapril maleate in patients of essential hypertension and diabetic hypertensives. International Journal of Basic \& Clinical Pharmacology. 2017 Jan 23;3(1):194-200 